\title{
El mundo integral y complejo del sujeto de la educación
}

\section{The subject of education's comprehensive and complex world}

Dr. Iván Valencia Martínez es Profesor Titular de tiempo completo en la Universidad del Atlántico (Colombia) (ivanvalencia09@hotmail.com) (https://orcid.org/0000-0002-5791-9830)

Dra. Cecilia Dolores Correa de Molina es Docente Titular de tiempo completo en la Universidad Simón Bolívar (Colombia) (ccorrea@unisimonbolivar.edu.co) (http://orcid.org/0000-0002-4862-6593)

Recibido: 2017-05-05 / Revisado: 2018-04-02 / Aceptado: 2018-05-07 / Publicado: 2018-07-01

\section{Resumen}

La adopción de un método etnográfico para la formación integral desde la complejidad centra su análisis en la educación, escenario significativo para interpretar e identificar nuevas formas de comprensión del acto educativo, donde la pedagogía y la didáctica admitida por el docente son fundamentales para religar los procesos académicos. Comprender desde la escuela que el sujeto de la educación es un ser humano multidimensional, sumergido en una diáspora de incertidumbres, certezas, dudas y utopías, situaciones que requieren miradas complejas para reconocer conexiones que dinamizan el tejido social del conocimiento. El método investigativo implementado, privilegia el análisis hermenéutico de los procesos que intervienen en la formación integral y asume su comprensión e interpretación en el contexto de la escuela, buscando penetrar en las simbologías e imaginarios de la comunidad durante del acto educativo. Para ello, la apropiación de un método etnográfico de investigación acción compleja para la transformación, constituye una vía metodológica emergente identificadora de realidades, para avanzar hacia procesos de integralidad, validar percepciones y reconceptualizaciones de la formación integral y generar actitudes de transformación en la comunidad docente y discente. Una práctica docente integral desde la complejidad propicia comprensión y valoración del estudiante en su condición de ser humano, otorga reconocimiento en el aula, necesario para un contexto social que reclama inclusión, dialogicidad y profunda sensibilidad ecológica y humana.

Descriptores: Método, etnografía, formación integral, complejidad, educación y praxis.

\begin{abstract}
The adoption of an ethnographic method for the comprehensive formation from the complexity centers it's object of analysis in the education, meaningul scenario to interpret and to identify new forms of grasping the educational act, in which pedagogy and teaching approach acknowledged by the educator are fundamental for the rebinding of the academic processes. Understanding from the school that the subject of education is a multidimensional human being, immersed in a plethora of uncertainties, certainties, doubts, hopes, pipe dreams, situations that
\end{abstract}

Forma sugerida de citar: Valencia, I., \& Correa de Molina, C. D. (2018). El mundo integral y complejo del sujeto de la educación. Alteridad, 13(2), 228-238. https://doi.org/10.17163/alt.v13n2.2018.06. 
require compound looks to recognize the connections that boosts the social fabric of knowledge. The investigation method implemented, favors hermeneutic analysis of the processes that take part in the comprehensive formation and takes on the understanding and interpretation in the context of the school, seeking to delve into the symbolism and imageries of the community during the educational act. For this purpose, the appropriation of an ethnographic method of research is a complex process for the transformation. it is an emerging methodological pathway that enables to identify realities, to progress towards

\section{Introducción}

El presente artículo da cuenta de los resultados de una investigación desarrollada en la ciudad de Barranquilla, cuyo propósito principal fue propiciar desde las concepciones emergentes de la complejidad, una praxis pedagógica docente contextualizada y transformadora de procesos de formación integral en el nivel básico secundario, a partir de la comprensión del estudiante en su condición de ser humano.

Investigación realizada teniendo en cuenta que, mover los cimientos disciplinares para suscitar las transformaciones y autotransformaciones que demanda la educación de cara a los compromisos formativos de los estudiantes, sugiere ir más allá del discurso, de la norma y de la ley; es decir, requiere de una discrecionalidad intersubjetiva del docente, expresada en procesos que exhorten la voluntariedad de la comunidad académica, si se tiene en cuenta la necesidad de resignificación de la concepción de escuela para remediar, por lo menos, sus contradicciones tanto internas como externas y repensarlas en un ambiente de sensibilidad (Hernández, 2014, p. 234) humana.

La formación integral desde la complejidad vislumbra nuevas posiciones de observación y construcción de teorías no sobre la educación, sino para la educación en relación sistémica con la formación y en la comprensión de otras lógicas en los campos problémicos que les toca abordar, para la explicación y acción didáctica de los procesos formativos en los diferentes niveles comprehensive processes, to validate perceptions and rethinking about comprehensive formation and to generate attitudes of transformation in the teaching community and the students alike. A comprehensive teaching practice from the complexity nurtures understanding and valuing the student in his human being condition, grants recognition in the classroom, necessary for a social context that demands inclusion, dialogue and profound ecological and human sensitivity.

Keywords: Method, ethnographic, comprehension formation, complexity, education, praxis.

donde interactúan. Ahora bien, formación y educación son categorías que conviven juntas, pero no son lo mismo y a pesar de hacer parte de una misma familia, entre ambas existen diferencias muy sutiles al decir de (Posada, 2008) "mientras la "formación" es una categoría más universal, que abarca al ser en su conjunto, la "educación" es menos universal, se refiere a la adquisición y desarrollo de elementos más focalizados (compartimientos, valores, conocimientos, competencias).

En este escenario, se propone una metodología etnográfica investigativa de acción compleja para la transformación, que busca no solo el reconocimiento de la multiplicidad de dimensiones que le son propias a los estudiantes en su condición de seres humanos, sino también, trasgredir las fronteras disciplinares para abordar una visión distinta, dinámica, dialéctica y sistémica, en la perspectiva de avanzar hacia una concepción transformadora y autotransformadora de la educación, con visión crítica, humana e inclusiva, al respecto: "Hay que reconocer las diversas dimensiones humanas y el compromiso que tenemos los docentes de desarrollar cada una de ellas. Como educadores, somos responsables del desarrollo de la dimensión cognitiva de nuestros estudiantes, pero tenemos iguales responsabilidades en la formación" (De Zubiría, 2013, p. 195).

Una concepción emergente de la complejidad implica comprender los problemas de la humanidad desde una perspectiva holística, donde lo unido, la incertidumbre, el caos, 
lo sistémico, lo transdisciplinar y lo complejo, serán referentes conceptuales que permitirán una mayor comprensión del Ser en el contexto de un mundo que al decir de Heisenberg (citado por Martínez, 2011b, p. 88): "parece un complicado tejido de acontecimientos en el que toda suerte de conexiones se alteran, se superponen o se combinan y de este modo determinan la textura del conjunto".

Apropiar metodológicamente desde el enfoque de la complejidad (Prigogine, \& Stengers, 2002) los problemas de la educación y de quienes actúan como sujetos de la misma, es hacer converger dialéctica, dialógica y sistémicamente diversas teorías y disciplinas con la finalidad de provocar que emerja un nuevo conocimiento que no tenga propiedad disciplinar.

Reconocer desde un enfoque complejo y transdisciplinar nuevos escenarios que permitan comprender integralmente los procesos sistémicos que dinamizan la educación-formación de los estudiantes en el marco de una escuela que propicie los procesos académicos desde un bucle articulador, es avanzar hacia otras opciones metodológicas que admitan la autotransformación y transformación del sujeto.

Es válido reconocer los esfuerzos que, en esta última perspectiva, ya han iniciado desde la escuela otras corrientes teóricas como el crítico social, la enseñanza problémica, pedagogía activa, pedagogía conceptual, aprendizaje sociocultural, entre otras, para buscar fundamentos y estrategias pedagógicas que sustenten la formación del Ser en el marco de una política que objetiviza la calidad educativa.

Ahora bien, la hermenéutica de la complejidad como opción metodológica desde la formación-educación, admite los saberes desde lo unido, en una dialéctica continua y discontinua, que hace del conocimiento un proceso integrador y diferenciador, en el cual al decir de Morín, citado por (González, 2008), "lo que está tejido en conjunto es un pensamiento que relaciona y se opone al aislamiento de los objetos de conocimiento..."...."lo complejo sería un elemento de la transformación, por lo que es necesario pensar la educación como una transformación del sujeto y de la sociedad misma."

Responder desde la escuela a los cambios culturales y avances científicos logrados desde las diversas áreas y disciplinas del conocimiento social y humano frente a la formación integral, donde los paradigmas, teorías y creencias vigentes empezaban a entrar en crisis, es reconocer un desarrollo parcial desde la disyunción, muy a pesar de que, desde ésta última, se obstaculiza la comprensión y formación integral del estudiante en su condición de Ser humano.

De esta manera, se refuta el modelo positivista y se apuntala hacia una metodología cualitativa emergente, donde la escuela reconozca al sujeto-objeto (dimensiones del estudiante) física, química y biológicamente por un lado, y psíquica, social y espiritualmente por el otro; como unidad y particularidad sistémica, en un contexto socioeconómicamente categorizado donde "alimento y cariño" (Martínez, 2009, p. 9), sean comprendidos sistémica y hologramáticamente articulados en un tejido cultural, con profunda sensibilidad humana, con particularidades definidas pero no definitivas, donde el todo es continuo y discontinuo en una permanente dialéctica de cambios cualitativos.

\section{Fundamentación teórica y metodológica integrada}

Una vía metodológica etnográfica de investigación acción compleja para la transformación (Valencia, 2016) no está basada en la mera y clásica descripción y análisis del fenómeno (observación pasiva, deliberación y especulación de las dimensiones), por el contrario, penetra al nivel de realidad (Nicolescu, 2011) que devela el contexto, para comprender, religar, sistematizar, validar integralmente los hallazgos, en un escenario de encuentros y desencuentros de imaginarios, simbologías, teniendo de presente una praxis que admita "el enfrentamiento de necesidades antagonistas, la creación de una realidad nueva 
en nosotros y fuera de nosotros, donde el imaginario en par-te se encarna transmuta, donde lo real se desgarra y se entreabre" (Morín, 2010, p. 37) para "aprender, desaprender y reaprender"
(Toffler, 1993, p. 271), en una acción dialéctica cualitativa que permitan una ininterrumpida reconceptualización del Ser en el contexto de su formación integral.

Gráfico 1. Representación de la categoría metodología etnográfica de investigación acción compleja para la transformación

\section{Formación integral y complejidad en la educación}

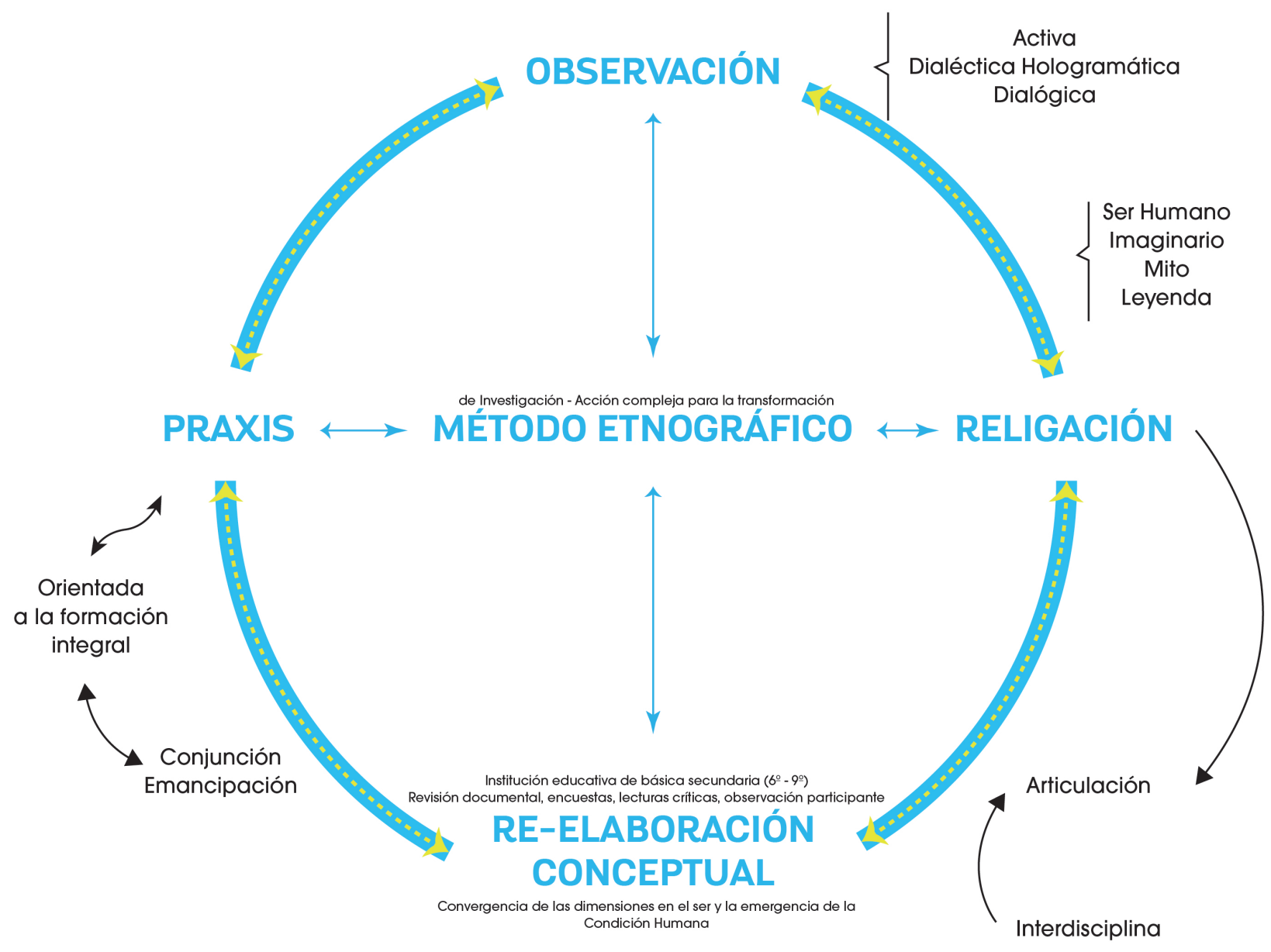

Fuente: Valencia Martínez, 2016.

Desde esta perspectiva de análisis, el observador se compenetra con la realidad develada, hace parte de ella, emitiendo y recibiendo recíprocamente influencias del objeto de indagación, en la perspectiva de no alcanzar principios de causalidad, ello implica al decir de Heisenberg que desde la incertidumbre, el "observador afecta y cambia la realidad que estudia" (Martínez, 2000, p. 16), por lo tanto ya no es posible llegar a principios y metas, sino generar actitudes de transformación en docentes y estudiantes desde las disciplinas y más allá de las disciplinas, iden- 
tificando una estructura compleja que metodológicamente asuma componentes asociados a observación activa; religación; deconstrucción y reconstrucción conceptual; praxis para la complejización de los procesos.

Esta categoría metodológica develada en el gráfico 1, sirvió como referente para la implementación y desarrollo de la tesis doctoral "Formación integral y complejidad en la educación básica secundaria" que además de articularse a los objetivos de la investigación, también fue identificando las estrategias de indagación de acuerdo con el contexto y a las circunstancias específicas que develaba la realidad estudiada. De importancia significativa para este proceso:

Un enfoque inicial exploratorio y de apertura mental ante el problema a investigar,(...) el uso de técnicas múltiples e intensivas de investigación con énfasis en la observación participante y en la entrevista con informadores representativos... lo cual admite un marco interpretativo que destaca el papel importante del conjunto de variables en su contexto natural para la determinación de la conducta, y que pone énfasis en la interrelación holística y ecológica de la conducta y los eventos dentro de un sistema funcional (Martínez, 2011, p. 200).

Ahora bien, la implementación de un método etnográfico de investigación acción compleja para la transformación, no descarta la apropiación de un enfoque cualitativo-fenomenológico, dado que:

Ofrece un punto de vista alterno y diferente del positivista acerca de la objetividad y los métodos apropiados para el estudio de la conducta humana. Básicamente, esta orientación sostiene que los científicos sociales no pueden comprender la conducta humana sin entender el marco interno de referencia desde el cual los sujetos interpretan sus pensamientos, sentimientos y acciones (Martínez, 2011, p. 201).

De esta manera, la formulación del método etnográfico de investigación-acción compleja para la transformación, permite comprender, complementar e "interpretar lo que ocurre desde el punto de vista de quienes actúan e interactúan en la situación problema" (Elliott, 1994, p. 25), además, de otros actores sociales presentes en el entorno próximo o lejano, lo que "ocurre", es necesario precisarlo, dado que "se hace inteligible al relacionarlos con los significados subjetivos que los participantes les adscriben. He ahí, porqué las entrevistas y la observación participante son importantes herramientas de investigación en un contexto de investigación-acción" (1994, p. 25).

En un contexto de investigación educativa compleja, su comunidad apropia y participa activamente del objeto de estudio e investigación, accede a la información, sistematización, interpretación y aprobación de los hallazgos, lo cual se desarrolla en un clima de confianza mutua "que solo puede ser validado a través del diálogo libre de trabas" (Elliott, 1994, p. 26).

Lo fundamental para un tipo de investigación etnográfica compleja desarrollada en un contexto escolar, es sensibilizar y consolidar un trabajo en equipo integrado por directivos, directivos docentes, profesores, estudiantes y padres de familia, capaces de oponerse, articularse y complementarse desde su rol de estudio profesional, frente a los problemas que hoy se le presentan al joven en un mundo planetario, dinamizado por un sistema de sistemas que desde lo lineal-disciplinar, lo tiende a deshumanizar, por consiguiente es necesario trabajar tanto con los contenidos disciplinares como con los contenidos no-disciplinares emergentes en las aulas, teniendo como foco principal el desarrollo humano (Batalloso y Moraes, 2012, p. 88).

Validar los procesos epistemológicos, teóricos y metodológicos desde una experiencia de indagación en el aula, propiciada desde un ambiente que facilite el proceso investigativo, es esencial para generar las actitudes de trasformación cognitiva y humana que demande una comunidad educativa. "La metodología que utiliza el profesor para enseñar...debe promover el desarrollo del pensamiento, que va desde la simple 
aproximación o demostración a la más compleja situación de aprendizaje" (Villalobos, 2001, p. 5).

\section{Análisis y resultados de la metodología implementada}

\subsection{Praxis compleja, generadora de transformaciones en la formación integral desde un contexto de aula}

La realidad o nivel de realidad que envuelve el mundo educativo en las organizaciones escolares revela un escenario diverso y rico en experiencias sociales y culturales que muchas veces no encuentran oportunidad de expresión en el aula, debido entre otras, a la presencia de códigos mentales e imaginarios dominantes que han sido legitimados desde el poder disciplinar.

La ruptura de fronteras entre las disciplinas (corolario de la multiplicidad de áreas científicas y de unos modelos de sociedad cada vez más abiertos, de la desaparición de barreras en la comunicación y de una mundialización de la información) viene obligando a la toma en consideración de modelos de análisis mucho más potentes que los que eran típicos de una única especialización disciplinaria.

La complejidad del mundo y de la cultura actual obliga a desentrañar los problemas con múltiples lentes, tantas como áreas de conocimiento existen; de lo contrario, es fácil que los resultados se vean afectados por las denominaciones que impone la selectividad de las perspectivas de análisis a las que recurre (Torres, 1998, pp. 29-95).

Avanzar metodológicamente hacia escenarios de aula que posibiliten la libre circulación y articulación de saberes interdisciplinares, constituyen una oportunidad para "entrelazar el conocimiento, ver todas las aristas posibles de construcción del mismo. Es ahí donde la transdisciplinariedad juega un importante rol en los procesos de investigación” (Nicolescu, 2002 en (González, 2009 , p. 14). La tendencia de una educación cognitiva disciplinar desarticulada de las disciplinas y de la formación, permite identificar al decir de (Correa, 2004a, p. 241) que "la concepción asignaturista y fragmentada del conocimiento, la parcelación entre la teoría y la práctica que han caracterizado los planes de estudios ha de ser superada por propuestas curriculares interdisciplinares".

No obstante, la escuela avanza de la mano de currículos disciplinares donde "el concepto tradicional de ciencia y su método empírico experimental, parecen, más bien, constreñir y aprisionar la imaginación creadora de nuestro pensamiento" (Martínez, 2011, p. 18), marcando límites, que aprisionan al Ser en un mundo que no es real, generando nuevas fronteras que impiden la diáspora de un pensamiento "que reconozca y trate los fenómenos multidimensionales en vez de aislar de manera mutiladora cada una de sus dimensiones... un pensamiento que aísla y separa hay que sustituirlo por uno que distinga y una" (Morín, 2000, p. 10).

Las disciplinas en el contexto de la sociedad del conocimiento alcanzan verdades parciales, no obstante y a pesar de ser verdades cortoplacistas, la evidente resistencia al cambio parece inquebrantable en el pensamiento docente, retar a la ciencia, sería a su juicio dar un paso a lo incierto, a lo desconocido, a lo oscuro, a la penumbra, generándose una tipología de miedos que invade el mundo objetivo y subjetivo de los seres humanos que hacen parte de esta comunidad, emerge el temor, la duda y desconfianza, es decir; el miedo a errar.

El miedo a errar en el aula es el miedo a perder el "poder" y "control" que otorga el conocimiento parcial de la disciplina, no hacerlo, es cederle espacio a otros saberes que circulan en el pensamiento del sujeto de la educación, muchas veces negados e incomprendidos, porque que escapan al saber y control disciplinar que posee el docente. Posibilitar el error en el aula implica desafiar la certeza positivista del conocimiento, es abrir diversas vías epistemológicas que interroguen la incertidumbre y hagan posible la emergencia de nuevas visiones críticos-sociales. Contexto que demanda una tipología de docentes con saberes 
integradores, que reten la disyunción y le apuesten a la conjunción susceptible de provocar una comprensión hologramática de los grandes desafíos que hoy retan a la educación: la formación integral.

La formación como categoría educativa ha sido escenario de controversia desde la antigüedad, pasando por los clásicos, contemporáneos y tiempos actuales, respectivamente se destacan teóricos de la talla de: Platón, Aristóteles, Humboldt, (Gadamer, 1999), (Guy, 2000), (Orozco,1999), (Tobón, 2010), (Nova, 2013) y (Correa, 2009), respectivamente entre otros, los cuales en su conjunto, han dejado valiosos aportes científicos que aún recrean y controvierten los diversos puntos de vista que cada campo conceptual sugiera.

Ahora bien, formar integralmente, es reconocer la existencia de una multidiversidad de dimensiones que están presentes en el ser humano. Ello implica, romper o acercar las fronteras que el mundo objetivo y subjetivo han creado para mantener incólume el principio cartesiano de la dispersión.

El docente debe reconocer que el desatrapamiento disciplinar, también es un proceso emancipador, le permite identificar que vivimos en un mundo caracterizado por "interconexiones a un nivel amplio y global” (Martínez, 2009, p. 20) lo cual implica, avanzar hacia la problematización e integración sistémica de saberes y conocimientos, al decir de (Bertalanffy, L. Von, y otros , 1981, p. 47), cuando expresa, "desde el átomo hasta la galaxia, vivimos en un mundo de sistemas".

Hacerlo, sugiere procesos de cambios a las formas de actuar, sentir, pensar y hacer; no obstante, resistencias aún vigentes en una tipología de docentes regidos por principios de inflexibilidad cognitiva, obstaculizan su religación, generándose un divorcio entre lo pensado y lo actuado, manteniéndose una especie de equilibrio "sin rumbo" que retrasa los procesos académicos y formativos esenciales desde una dialéctica cualitativa.

La transformación y la autotransformación son actos sistémicos propios de la individualidad del Ser, donde intervienen aspectos sociales, cultu- rales y genéticos, por consiguiente, no la propicia la norma, ley, reglamento y/o capacitaciones, sino la misma persona. En este sentido, la historia registra la asistencia masiva de docentes a encuentros de capacitación y formación, justificadas muchas veces por un sinnúmero de razones que van desde las más exóticas hasta las más inverosímiles, convirtiendo esos escenarios en espacios de dialogo sustentados desde acciones rutinarias y mecanizadas que una vez regresan al aula, divorcian rápidamente la teoría de la práctica y viceversa.

Ahora bien, no se trata de ir al extremo, e invalidar o negar que la existencia de estrategias pedagógicas de cualificación docente aún tengan una vigencia conceptual en la escuela y fuera de ella, favoreciendo experiencias positivas para el cumplimiento de las metas que consensualmente acuerda la planeación institucional; de lo que se trata, es identificar desde la investigación, una estrategia metodológica de pertinencia conceptual y contextual, que provoque desequilibrio epistemológico en la comunidad docente y estudiantil, es decir, provocar cambios de actitud evidentes en una praxis sistémica entre la cognición y la formación integral.

\subsection{Aportes teóricos y metodológicos desde la complejidad a la forma- ción integral}

El mundo académico transita de la mano de un sinnúmero de categorías que muchas veces encuentran un nivel parcial de comprensión e interpretación desde la ciencia positivista, no obstante ese mundo está inmerso en un entretejido dialéctico de ideas, pasiones, frustraciones, esperanzas, contradicciones, incertidumbres, cegueras, sentires, que transitan individual y colectivamente en la objetividad y subjetividad del ser humano, los cuales difícilmente pueden ser analizadas desde el filtro de la disyuncionalidad, superar ese obstáculo teórico y metodológico, requiere de visiones integradoras capaces de reconocer el todo en las partes y las partes en su relación sistémica con el todo, es decir, apropiar procesos religantes de un pensamiento hologramático y complejo. 
El ser humano por naturaleza es complejo, una visión disciplinar solo ilumina una parte de la penumbra cognitiva, una visión inter y transdisciplinar abre una diáspora de interconexiones que aproximan la comprensión e interpretación de la realidad en la cual se sumerge el mundo integral del estudiante, donde "la transdisciplinariedad sería un conocimiento superior emergente, fruto de un movimiento dialéctico de retro- y pro-alimentación del pensamiento" (Martínez, 2011a, p. 67).

No obstante, adoptar una investigación etnográfica con visión compleja en un terreno de dominio y control disciplinar, es abrir una batalla simbólica de ideas, conceptos, percepciones e imaginarios, es luchar contra esquemas mentales que durante mucho tiempo han permanecido como protectores de la inalterabilidad y de la certeza que produce la cientificidad, caracterizadas por exhibir una marcada resistencia hacia el cambio, la transformación y la incertidumbre.

Una metodología etnográfica de investigación acción compleja para la transformación, es una experiencia de campo innovadora, significa retar desde el aula la implacable resistencia docente provocada por la dependencia disciplinar. No es el registro de voces, formas de actuar, pensar, vestir, lo que pasivamente debe consignar el diario de campo, máxime cuando la presencia de un "extraño"; es decir, del investigador, altera el clima organizacional y provoca una especie de "prevención", entre otras, porque los resultados de una investigación que indaga pedagogía y didáctica docente pueden comprometer el encargo social encomendado.

Sin alterar la ética de la información y a la luz del consenso avalado desde las organizaciones escolares, una metodología etnográfica de investigación acción compleja para la transformación, contempla la implementación de diversas estrategias pedagógicas y didácticas que ganen pertinencia contextual al momento de generar desde sus propias voces desequilibrios epistemológicos en una comunidad educativa.

Las voces y percepciones emitidas por los actores claves de la comunidad educativa, al ser llevadas al aula de clases o al escenario institucional de convergencia docente, pueden generar una especie de resonancia epistemológica que provoca de acuerdo a la actitud, reflexión, angustia, impotencia o transformación; es decir, poner a dialogar las voces de los interlocutores al mismo tiempo que desarrollan el acto educativo, propicia un ambiente de análisis reciproco frente a las particulares formas de pensar, sentir y actuar del docente y del discente.

Ello produce una especie de "negociación" en el mejor sentido del término entre la realidad develada y el mundo objetivo y subjetivo docente y discente, expresada en un cambio de actitud visiblemente validado y refrendado por la propia comunidad educativa.

La intención cualitativa se expresa al consensuar una apropiación pedagógica y didáctica más integradora con sensibilidad y afectividad, desencadenantes de un ambiente escolar que propicia mejores aprendizajes, en un escenario de aula que asume su propia autoevaluación articulada a los procesos misionales de la comunidad educativa, desde una dinámica hologramática, comprensiva, interpretativa y valorativa del estudiante en su condición de ser humano para "reconciliar los puntos de vista de acuerdo con principios y valores" (D’Angelo, 2005, p. 159).

La valoración, reconocimiento e inserción de los saberes populares que acompañan la cotidianidad de los estudiantes, y la articulación sistémica de saberes entre disciplinas al momento de desarrollar las clases, propician autoestima, responsabilidad, seguridad y participación en los estudiantes, haciendo posible la emergencia de estas nuevas unidades de análisis, propias de una escuela con pertinencia conceptual y contextual.

El aula de clases se convierte en un espacio agradable para la interlocución de saberes y conocimientos, de esta manera, los docentes admiten reconocer que sus estudiantes también son seres humanos integrados por una multiplicidad de dimensiones interconectadas entre sí.

La innovación pedagógica y didáctica es fundamental para estimular los aprendizajes diferenciadores, propios de aquellos escenarios 
de aula que reconocen todos los puntos de vista como sinónimos del consenso y disenso, garantes de una circulación dialéctica de ideas, pensamientos y conocimientos.

La escuela en su conjunto por naturaleza es dialéctica, devela muchos rostros, muchas tensiones, propia de una dinámica humana sometida permanentemente a desequilibrios cognitivos y formativos. Esta velocidad que mueve el conocimiento pone a riesgo la linealidad de la "verdad"; el mundo es complejo, cambiante, sistémico, abierto a saberes emergentes, articulados a un escenario de aula donde la duda, la pregunta, la incertidumbre, constituyen retos permanentes para el docente y la escuela.

Premisas estructurales para considerar que la institución escolar debe propiciar escenarios de cualificación docente en procesos de formación integral desde la complejidad, con la finalidad de entretejer saberes interdisciplinares que amplíen visiones cognitivas, formativas, integrativas y humanas.

Ese contexto de realidades advierte la necesidad de dar inicio a una reforma curricular integradora de saberes con visión holística (Guyas Fitch et al., 2015), propia de instituciones que hayan identificado el plus diferenciador asociado a la multiplicidad de saberes. Vincular la comunidad docente a procesos de integralidad cognitiva y humana, con la intención de cultivar una nueva cultura pedagógica que coherente con la apropiación de nuevas didácticas, puedan hacer del modelo pedagógico una oportunidad dialógica y multidimensional. Realidad emergente que propicia condiciones para entrelazar el intangible socio-cultural presente en la unidad familiar a los procesos de complementariedad cognitiva y formativa de la escuela.

Escenario para acentuar la cultura de la participación multidiversa desde la promoción y el reconocimiento del otro, teniendo en cuenta, valores, emociones, habilidades y competencias, perspectiva sugestiva para estimular procesos permanentes de autoevaluación desde el aula, mediados por las voces de los participantes sujetos de la educación.
La educación es un entretejido sistémico de saberes y conocimientos sociales y humanos, son múltiples y entrelazadas las percepciones que llegan al estudiante, razón para considerar necesario que, desde el aula, los docentes mantengan visiones integrales independientemente del nivel o grado educativo donde se encuentre el sujeto de la educación.

Superar las resistencias objetivas y subjetivas, constituyen un desafío estructural para el docente, liberar el pensamiento lineal y avanzar hacia pensamientos y saberes interdisciplinares, es dar un salto cualitativo hacia la emancipación del Ser humano. Por ello, la actitud de cambio y transformación es un acto individual que requiere una profunda y decidida articulación entre el pensar, el sentir y el actuar. Ello implica reconocer sus propias dimensiones y las que le son propias al mundo objetivo. Solo así aproximaríamos una vía para avanzar hacia un proceso de comprensión del mundo integral y complejo del sujeto de la educación.

\section{Conclusiones}

La dispersión disciplinar en una limitante epistemológica para el conocimiento integral. La ignorancia de saberes interdisciplinares produce efectos nocivos para la formación refrendada en una tipología de aula donde el docente apropia discrecionalmente una estrategia selectiva de interlocución en temas relacionados con la disciplina, sesgando la intervención colectiva y propiciando otro tipo de dispersión que afecta la libertad y cercena la circulación de saberes diferenciadores.

El poder del saber disciplinar es el poder del docente en el aula, produce ceguera interdisciplinar, los estudiantes globalizan sus interrogantes, lanzan señales de inconformidad que muchas veces el docente no escucha, su prioridad está centrada en el "cumplimiento" del tema y del programa, visibilizar esas percepciones en el aula, produce desequilibrios metodológicos y didácticos siempre y cuando medie una disposición individual de cambio y transformación.

Liberarse del poder disciplinar es alcanzar desde la educación la emancipación de las ideas, 
avanzar hacia la autonomía del pensamiento, al decir de (Correa, 2004b, p. 61) significa que "la educación ha de entenderse como una influencia no manipuladora de los estudiantes, docentes, padres y madres de familia: su meta será contribuir a que las personas desarrollen su autonomía, principio fundamental de la formación integral".

Mediar con los códigos intersubjetivos de estudiantes y docentes significa culturalmente intervenir los imaginarios, ideologías, miedos y resistencias, que acompañan la individualidad del Ser, es decir, disponerlos culturalmente al cambio y a la transformación mental, necesaria para avanzar hacia una nueva experiencia conceptual de la escuela:

(...) desde allí podrá disponerse una profunda reflexión, un gran acontecimiento reflexivo con cada uno de tales aspectos, pues, quizás, se pueda iniciar el planteamiento de las tramas para una Educación Básica con posibilidad de recurrir, desmedidamente, a otro modo de pensar, de vivir, de ser (Peñalver 2003, p. 3).

Se requiere dinamizar cambios y transformación desde el aula, "su misión no es el traspaso de contenidos y saberes sino más bien, dotar a sus estudiantes de capacidades para progresar y ordenar lo aprendido: aprender a aprender"(Fuentes, 2011, p. 6) susceptibles de ser jalonadas por corrientes intelectuales no atadas al formalismo normativo, y hagan de la escuela un laboratorio pedagógico y didáctico que favorezca la realización del sueño de concebir la educación como un proceso sistémico complejo de formación integral con profunda sensibilidad e integralidad humana y ecológica, en el contexto de una escuela que reconozca y articule la multidimensionalidad del estudiante como ser humano, protagonista de saberes y aprendizajes pertinentes en el contexto de una realidad emergente.

\section{Referencias bibliográficas}

Basarab Nicolescu (2011). La necesidad de la transdisciplinariedad en la educación superior.
Discurso central del Congreso internacional de educación superior celebrado en Estambul, Turquía 27-29 de mayo.

Batalloso, J., \& Moraes, C. (2012). ¿Qué es una escuela transdisciplinar? En González Velazco, Teoría Educativa Transcompleja Tomo I. Bogotá: Ediciones Universidad Simón Bolívar.

Bertalanffy, L. Von, y otros (1981) Historia y situación de la teoría general de sistemas. Tendencias en la teoría general de sistemas. Madrid: Alianza.

Constitución Política de Colombia (1991).

Correa, C. (2004a). Gestión y evaluación de calidad. Bogotá: Cooperativa Editorial Magisterio.

Correa, C. (2004b). Currículo dialógico, sistémico $e$ interdisciplinar. Bogotá: Transversales Magisterio.

Correa, C. (2009). Currículo, inclusividad y cultura de la certificación. Barranquilla: Ediciones la Mancha del Quijote.

D’Angelo, O. (2005). Autonomía integradora y transformación social: El desafío ético emancipatorio de la complejidad. La Habana: Editorial Linotipia Bolívar.

De Zubiría Samper, J. (2013). El maestro y los desafíos a la educación en el siglo XXI. REDIPE Virtual 825 , julio. ISSN 22561536. Recuperado de https://goo.gl/2xFX6F

Elliott, J. (1994) La investigación-acción en educación. Madrid: Ediciones Morata.

Fuentes, X. V. (2011). Reflexión en torno a la gestión de aula y a la mejora en los procesos. Revista Iberoamericana de Educación, 55(3), 1-7.

Gadamer, H. (1999). Verdad y Método I. Fundamentos de una hermenéutica filosófica. Salamanca: Editorial Sígueme.

González, J. (2008). Investigando el propio accionar educativo en el contexto del pensamiento complejo. Revista Integra Educativa, 1(1), 109-120.

Guyas Fitch, R. I.; Esparza Parga, R.; Romero Sánchez, M. C.; Rubio Barrios, J. E. (2015). Modelo de Educación Holística: Una propuesta para la formación del ser humano. Actualidades Investigativas en Educación, 15(3), 1-25.

https://doi.org/http://dx.doi.org/10.15517/aie.v15i3.20654

Hernández, L.J. (2014). La pedagogía de la sensibilidad y los acercamientos al sujeto descentrado. Educere, 18(60), 229-236. Mérida: Universidad de los Andes. 
Martínez, M. (2000). La investigación cualitativa etnográfica en educación. México: Editorial Trillas.

Martínez, M. (2009). Nuevos paradigmas en la investigación. Caracas: Editorial ALFA.

Martínez, M. (2011). Comportamiento humano. México: Editorial Trillas.

Martínez, M. (2011a). Paradigmas emergentes y ciencias de la complejidad. Revista Opción, 27(65), 45-80.

Martínez, M. (2011b). Tesis doctoral I V M, p. 20.

Morín, E. (2000). La mente bien ordenada. Repensar la reforma. Reformar el pensamiento. Barcelona: Seix Barral.

Morín, E. (2010). A favor y en contra de Marx. Tucumán: Nueva Visión.

Nicolescu, B. \& Cols. (2002). Educação e Transdisciplinaridade. Brasília: USP.

Nova, A. (2013). Políticas para la formación integral en la educación superior en Colombia. Tunja: Universidad Pedagógica y Tecnológica de Colombia, Memorias del Congreso Educación y Pedagogía.

Orozco, L. (1999). La formación integral: mito y realidad. Bogotá: Universidad de los Andes.

Posada Álvarez, R. (2008). Competencias, currículo y aprendizaje en la formación superior. Barranquilla: Universidad de Atlántico.

Prigogine, I. \& Stengers, I. (2002). La nueva alianza: metamorfosis de la ciencia. Barcelona: Alianza.

Tobón, S. (2010) Formación integral y competencias. Pensamiento complejo, currículo, didáctica y evaluación, Bogotá: ECOE

Toffler, A. (1995). El shock del futuro. Barcelona: Plaza y Janés.

Torres Santomé, J. (1998). Las razones del curriculum integrado. Madrid: Morata.

Valencia, I. (2016) Formación integral y complejidad en la educación básica secundaria. (tesis doctoral). Universidad Simón Bolivar, Barranquilla, Colombia. 